CLINICAL STUDY

\title{
Progression of medullary thyroid carcinoma: assessment with calcitonin and carcinoembryonic antigen doubling times
}

\author{
Anne Laure Giraudet ${ }^{1}$, Abir Al Ghulzan ${ }^{2}$, Anne Aupérin ${ }^{4}$, Sophie Leboulleux ${ }^{1}$, Ahmed Chehboun ${ }^{1}$, \\ Frédéric Troalen ${ }^{2}$, Clarisse Dromain ${ }^{3}$, Jean Lumbroso ${ }^{1}$, Eric Baudin ${ }^{1}$ and Martin Schlumberger ${ }^{1}$ \\ Departments of ${ }^{1}$ Nuclear Medicine and Endocrine Oncology, ${ }^{2}$ Biopathology, ${ }^{3}$ Radiology and ${ }^{4}$ Biostatistics and Epidemiology, Institut Gustave Roussy and \\ University Paris Sud, 94805 Villejuif Cédex, France \\ (Correspondence should be addressed to M Schlumberger; Email: schlumbg@igr.fr)
}

\begin{abstract}
Objective: The progression of medullary thyroid cancer is difficult to assess with imaging modalities; we studied the interest of calcitonin and carcinoembryonic antigen (CEA) doubling times and of Ki-67 labeling and mitotic index (MI).

Patients and methods: Fifty-five consecutive medullary thyroid carcinoma (MTC) patients with elevated calcitonin levels underwent repeated imaging studies in order to assess tumor burden and progression status. We looked for relationships between tumor burden and levels of calcitonin and CEA and between progression status according to the response evaluation criteria in solid tumors (RECIST) and calcitonin and CEA doubling times, and Ki-67 labeling and MI.

Results: The calcitonin and CEA levels were correlated with tumor burden. Ten patients with calcitonin levels below $816 \mathrm{pg} / \mathrm{ml}$ had no imaged tumor foci. Among the 45 patients with imaged tumor foci, 19 had stable disease and 26 had progressive disease, according to the RECIST. The calcitonin and CEA doubling times were strongly related to disease progression, with very few overlaps: $94 \%$ of patients with doubling times shorter than 25 months had progressive disease and $86 \%$ of patients with doubling times longer than 24 months had stable disease. Ki-67 labeling and MI were not significantly associated with disease progression.

Conclusion: For MTC patients, the doubling times of both calcitonin and CEA are efficient tools for assessing tumor progression.
\end{abstract}

European Journal of Endocrinology 158 239-246

\section{Introduction}

Medullary thyroid carcinoma (MTC) develops from thyroid $\mathrm{C}$ cells that produce calcitonin. Calcitonin is a sensitive and specific marker of the disease and remains detectable in half of the patients after surgery for clinical disease (1). Clinical recurrences are often found at an early stage because elevated calcitonin levels lead to their compulsive search, and then disease will usually progress slowly with time. Survival after recurrence may, thus, extend over decades (2). Indeed, as for any tumor, prognosis is related to both tumor burden and progression rate (3). Assessing these two characteristics in the MTC patients will permit the few patients with an aggressive disease to select for new treatment modalities and to interpret stable disease during treatment (4).

Assessment of tumor burden requires a combination of multiple imaging modalities because metastases in the MTC patients often involve multiple organs and are often multiple in each involved organ, as recently reported on the present series of patients (5). Assessment of the progression status according to the response evaluation criteria in solid tumors (RECIST) (6) in these often slowly progressive tumors may be complicated, even if imaging modalities are standardized. Therefore, we sought other indicators of disease progression.

In a given patient, serum calcitonin and carcinoembryonic antigen (CEA) levels are related to tumor burden (7), though production of these markers may differ between tumors and tumors with low expression of calcitonin and higher production of CEA may be more aggressive (8-10). The trend of the calcitonin and CEA levels can be quantified by their doubling times (11-14). Circulating calcitonin levels may vary by $30 \%$ or even more at short intervals of time. However, because no effective treatment modality was available, many patients even with metastatic disease did not receive any treatment for long periods of time extending over years, and this allowed accurate determination of the calcitonin and CEA doubling times. The doubling times have been related to various clinical outcomes, such as survival rate, time to recurrence, and time between recurrence and death $(11,13)$, but so far no relationship has been reported with the progression status assessed on imaging modalities using the RECIST. 
Tumor growth depends on mitotic rate, which can be estimated by mitotic index (MI) and Ki-67 labeling on tumor tissue samples, as already demonstrated in many other primary tumors, including other thyroid cancer types $(15,16)$. It has been suggested that Ki- 67 may be a prognostic indicator in MTC (17).

Finally, survival in many other primary tumors is related to the level of 2-[fluorine-18]-fluoro-2-deoxy-Dglucose (FDG) uptake (18-23). A correlation between FDG uptake and Ki-67 expression was found in MTC patients (24). However, FDG uptake in MTC patients is usually low and cannot be used to predict the outcome of individual patients $(5,25)$.

In the present study on 55 consecutive MTC patients followed at Institut Gustave Roussy, we first looked for relationships between serum calcitonin and CEA levels and tumor burden. Then we assessed the progression status according to the RECIST by comparing standardized imaging modalities performed at a 1-year interval and studied one relationships with circulating calcitonin and CEA doubling times and Ki-67 labeling index on tumor samples.

\section{Patients and methods}

\section{Patients}

The 55 consecutive MTC patients followed at Institut Gustave Roussy, who had elevated plasma calcitonin levels, were included in the study from January 2005 to August 2006.

Patients had been followed up every 4-12 months since initial treatment and at each visit had calcitonin and CEA determinations. For each patient, all the calcitonin and CEA levels and the day of determination since initial treatment were recorded from the clinical chart. We also recorded all treatment modalities, the date of discovery of a clinical recurrence and its site, and the clinical status at the end of follow-up.

At inclusion in the present study, a complete imaging workup was performed with standardized procedures and included a neck and liver ultrasonography; a bone scintigraphy; a neck, chest, and abdomen spiral computed tomography (CT) scan; a bone and liver magnetic resonance imaging; and an FDG positron emission tomography (PET) scan (5). This permitted an accurate estimation of the tumor burden in each patient. In the 45 patients with demonstrated lesions, the comparison of imaging modalities performed at an interval of 1 year permitted classification of disease as either progressive, in the case of appearance of new lesions or of an increase by $20 \%$ or more of the sum of the diameters of the target lesions, or stable disease, according to the RECIST (6). No treatment was given during this study period.

The protocol was approved by the scientific review committee of the Institut Gustave Roussy and the ethical committee of Bicêtre. Informed consent was obtained from each patient for the studies on tumor samples.

\section{Methods}

Calcitonin and CEA levels and doubling times Plasma calcitonin level has been measured since 1983 using the ELSA-CT kit (Cis-Bio International, Gif-surYvette, France) functional sensitivity was improved from 10 to $4 \mathrm{pg} / \mathrm{ml}$ in 2002; normal range $<10 \mathrm{pg} / \mathrm{ml}$. This is an IRMA method using two monoclonal antibodies, which are highly specific for calcitonin with no crossreaction with procalcitonin (26). The standard curve $(0-500 \mathrm{pg} / \mathrm{ml})$ is linear from 10 to $500 \mathrm{pg} / \mathrm{ml}$ and dilutions were performed for values above $400 \mathrm{pg} / \mathrm{ml}$.

CEA was measured using the Enzymun-test CEA kit (Boehringer, Mannheim, Germany; normal $<7 \mathrm{ng} / \mathrm{ml}$ ).

To assess the doubling time of calcitonin and CEA, all determinations were taken into account from 6 to 12 months after initial treatment and until any major therapy was undertaken for a total of 14-240 months (median 78 months). After logarithmic transformation, the calcitonin and CEA levels were linearly related to time, indicating either an exponential increase or a stable level during the study period. To exclude artifacts due to errors in measurement or short-term spontaneous variations, the doubling time was calculated only when at least four measurements were linearly related to a correlation coefficient higher than 0.85 . The doubling time was then calculated as the time necessary for an increase in the calcitonin or CEA levels by natural logarithm of $2(\ln (2))$ (Fig. 1).

MI and Ki-67 immunostaining The MI corresponded to the number of mitoses observed in the nuclei on a surface of $2 \mathrm{~mm}^{2}$ (successive fields) with a $400 \times$ magnification in the area with the highest mitotic count. The MI was assessed on histological sections stained by hematein, eosin, and saffran.

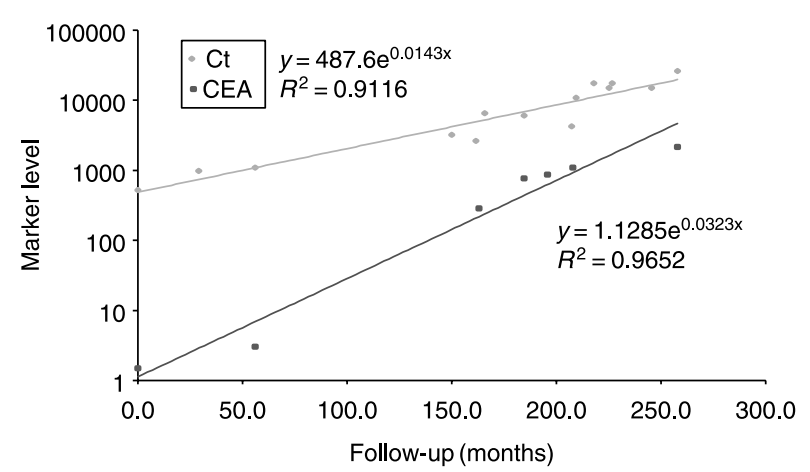

Figure 1 Plasma calcitonin (Ct) and serum CEA levels in a patient with metastatic MTC followed for 22 years, during which no systemic treatment was given. Note that after logarithmic transformation, there is a linear increase in both calcitonin and CEA levels, in relation to an exponential increase in tumor masses. These data allowed an accurate determination of calcitonin and CEA doubling times. 
For Ki-67 immunostaining, the antigen retrieval was performed by incubating the tissue sections three times for $5 \mathrm{~min}$ in phosphate buffer $(\mathrm{pH} 7.3)$ in $850 \mathrm{~W}$ microwaves. Tissue sections were then incubated for $1 \mathrm{~h}$ with the anti-Ki-67 MAB (Clone MIB1, Dako M7240, Dako A/S, Glostrup, Denmark) at a 1:75 dilution. The revelation of the staining was performed using the kit DAKO CSA K.1497, and diaminobenzidine (Dako A/S, Glostrup, Denmark) as the chromogen. MIB1 staining was assessed by estimating at a $200 \times$ magnification the percentage of positive neoplastic nuclei within the area of highest positivity at low power $(100 \times$ magnification). A total of 500 nuclei were examined. All homogeneously stained nuclei, even with a light staining or with only a nucleolar staining, were considered positive.

FDG PET scan FDG PET scan was performed on an integrated PET-CT Biograph LSO system (Siemens Medical Solutions, Erlangen, Germany) after the i.v. injection of $5 \mathrm{MBq} / \mathrm{kg}$ of FDG, followed by a $60-\mathrm{min}$ uptake phase. Standardized uptake value (SUV) was calculated for each tumor focus larger than $1 \mathrm{~cm}$ in diameter. $\mathrm{SUV}_{\max }$ was extracted automatically using e.Soft tools (Siemens Medical Solutions). In each patient, the highest $S_{U} V_{\text {max }}$ was taken into account for statistical analyses. Among the 45 patients with imaged tumor foci, no abnormal FDG uptake was found in 15 patients; in the remaining 30 patients, $\mathrm{SUV}_{\max }$ was relatively low, being equal to or $<2$ in 6 patients, ranging from 3 to 4 in 16 , and from 5 to 6 in the other 8 patients.

\section{Statistical analysis}

Median and range of quantitative variables are presented. Quantitative variables were compared between the different groups defined by disease extension and progression status using Kruskal-Wallis rank test. Correlations between quantitative variables were analyzed by the Kendall $\tau$ rank correlation coefficient.

Ki-67 labeling and $\mathrm{MI}$ indexes and $\mathrm{SUV}_{\max }$ were analyzed as quantitative variables and were also dichotomized in two classes defined by a threshold equal to the median value. For the calcitonin and CEA doubling times, the threshold was 24 months; this value was close to the median value and was used in previous publications $(11,13)$.

\section{Results}

\section{Patients}

Fifty-five consecutive MTC patients were enrolled in the study, of whom 21 were women and 34 were men, with a median age of 56 years (range 14-83 years). Of these patients, 5 had a multiple endocrine neoplasia (MEN) $2 \mathrm{~A}$ syndrome, 3 had a MEN 2B syndrome, 4 had a familial
MTC, and the remaining 43 had a sporadic MTC. According to the 2002 pathological tumor-node-metastases (pTNM) classification (27), thyroid tumor was classified as pT1 in 5 patients, pT2 in 14, pT3 in 11, pT4a in 12, pT4b in 9, and pTx in 4; as pNO in 4 patients and pN1 in 51; and as M1 in 14 patients (in bones in 7 patients, in lungs in 5, and in liver in 8). The median follow-up after initial surgery was 79 months (range 3-309 months), during which 31 patients without initial distant metastases experienced a clinical recurrence. The time between initial treatment and recurrence ranged from 3 to 192 months (median 62 months).

At the time of the study, ten patients had no imaged lesion. The remaining 45 patients had demonstrable tumor foci; in the neck in 33 patients, in the mediastinum in 17 , in the lungs in 19 , in the liver in 25 , in bones in 25 , and at other sites in 4 . Lesions were located only in the neck or mediastinum in ten patients (in the neck only in eight patients, in the mediastinum only in one patient, and in the neck and mediastinum in one patient) and only at distant sites in nine patients (at one metastatic site in four patients and at two to four metastatic sites in five patients). In the remaining 26 patients, the neck and mediastinum involvement was associated with distant metastases: at one distant site in 7 patients and at two to four distant sites in 19 patients.

In these 45 patients with lesions demonstrated on the first imaging workup at inclusion in the study, comparison of imaging modalities performed at a 1-year interval according to the RECIST showed that 19 patients had stable disease and 26 had progressive disease.

\section{Calcitonin and CEA levels}

At the time of the study, plasma calcitonin levels ranged from 21 to $247000 \mathrm{pg} / \mathrm{ml}$ (median $1250 \mathrm{pg} / \mathrm{ml}$ ) and CEA levels ranged from 1 to $5845 \mathrm{ng} / \mathrm{ml}$ (median $37 \mathrm{ng} / \mathrm{ml}$ ). The calcitonin and CEA levels were related to the number of imaged tumor sites (Fig. 2). In the ten patients with no imaged lesion, the median calcitonin level was $196 \mathrm{pg} / \mathrm{ml}$ (range 39-816 pg/ml), and the median CEA level was $11 \mathrm{ng} / \mathrm{ml}$ (range $1-33 \mathrm{ng} / \mathrm{ml}$ ). In the ten patients who had only a neck or mediastinum recurrence, the median calcitonin level was $565 \mathrm{pg} / \mathrm{ml}$ (range 191-1440 pg/ml), and the median CEA level was $12 \mathrm{ng} / \mathrm{ml}$ (range $1-71 \mathrm{ng} / \mathrm{ml})$. In the 11 patients who had one distant metastatic site, the median calcitonin level was $1510 \mathrm{pg} / \mathrm{ml}$ (range $21-6000 \mathrm{pg} / \mathrm{ml}$ ), and the median CEA level was $37 \mathrm{ng} / \mathrm{ml}$ (range $2-927 \mathrm{ng} / \mathrm{ml}$ ). In the 24 patients who had two to four distant metastatic sites, the median calcitonin level was $18450 \mathrm{pg} / \mathrm{ml}$ (range 276-247 $000 \mathrm{pg} / \mathrm{ml}$ ), and the median CEA level was $531 \mathrm{ng} / \mathrm{ml}$ (range 4-5845 ng/ml). Although the circulating calcitonin and CEA levels were highly different between these four groups of patients with different tumor burdens $(P<0.0001$ and $P<0.0001)$, there was a large overlap in the calcitonin and CEA levels between these groups. 
(a) Calcitonin according to the number of tumor sites

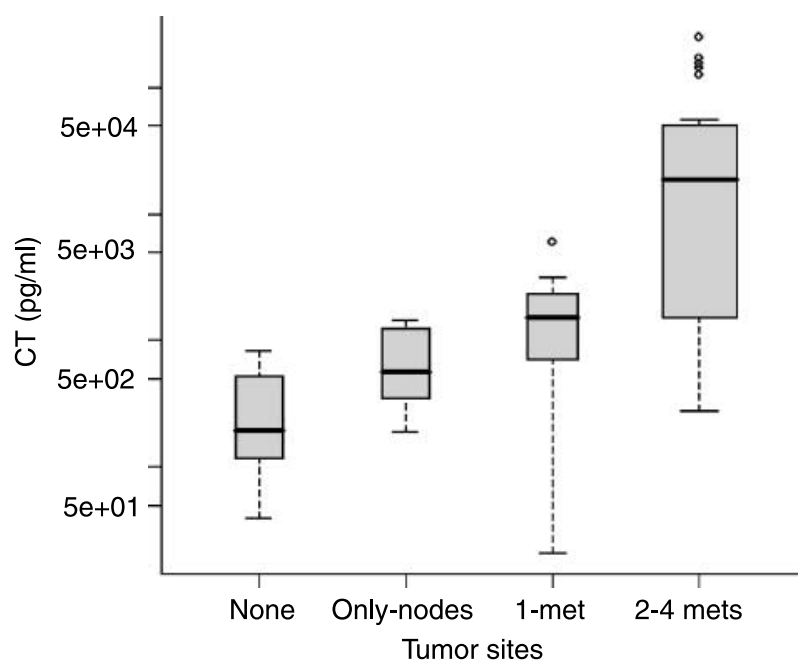

(b)

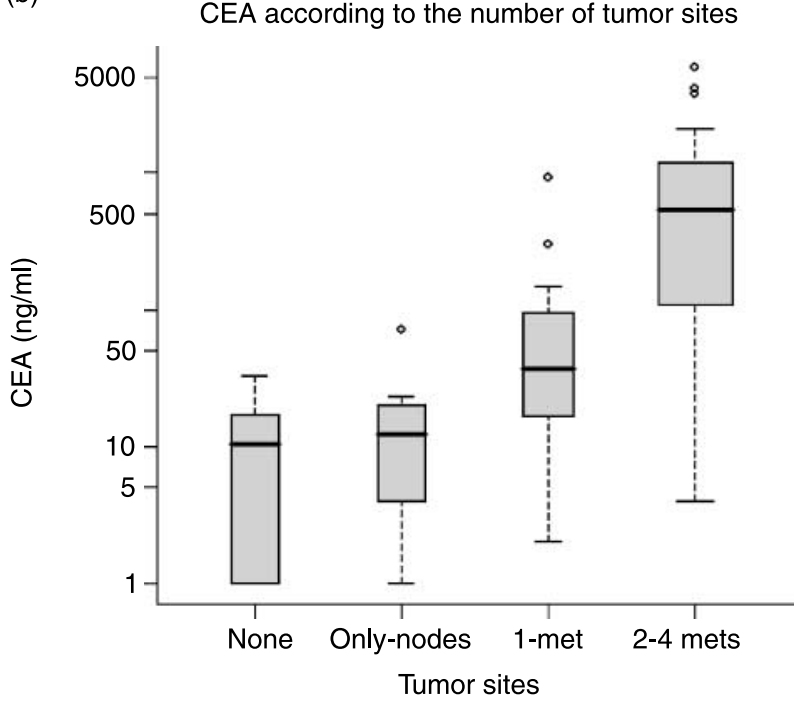

Figure 2 (a) Plasma calcitonin level according to the number of tumor sites. The levels of calcitonin are represented by box plots in each class defined by disease extent; logarithmic scale is used. The edges of the box are the 25th and 75th percentiles and the line in the box is the median. The ends of the vertical lines or 'whiskers' indicate the minimum and maximum data values; unless outliers (noted with circle) are present in which case the whiskers extended to a maximum of 1.5 times the inter-quartile range. No imaged site $n=10$; only neck or mediastinum nodes $n=10 ; 1$ metastatic site $n=11 ; 2-4$ metastatic sites $n=24$. Calcitonin level increased significantly with disease extent $(P<0.0001)$. (b) Serum CEA level according to the number of tumor sites. The levels of CEA are represented by box plots in each class defined by disease extent; logarithmic scale is used. The edges of the box are the 25th and 75 th percentiles and the line in the box is the median. The ends of the vertical lines or 'whiskers' indicate the minimum and maximum data values; unless outliers (noted with circle) are present in which case the whiskers extended to a maximum of 1.5 times the interquartile range. No imaged site $n=10$; only neck or mediastinum nodes $n=10 ; 1$ metastatic site $n=11 ; 2-4$ metastatic sites $n=24$. CEA level increased significantly with disease extent $(P<0.0001)$.

\section{Calcitonin and CEA doubling times}

A total of 4-45 determinations (median 14 determinations) were available per patient. The calcitonin and CEA doubling times could not be accurately determined in three or four patients.

The calcitonin and CEA doubling times were highly variable among patients and ranged from 2 to 146 months. The median calcitonin and CEA doubling times were 19 and 25 months respectively. In a given patient, the calcitonin doubling time was not related to the calcitonin and CEA levels, and did not vary significantly during follow-up (Fig. 1). They were not related to the locations of metastatic foci or the number of involved sites. The doubling times were calculated using measurements performed before any therapeutic intervention, which might have decreased both the calcitonin and CEA levels. It is interesting to note that the doubling times calculated before and after therapeutic interventions were similar.

The calcitonin doubling time was less than or equal to 6 months in 6 patients, ranged from 7 to 24 months in 22 , and was longer than 24 months in 24 , among whom it was longer than 60 months in 11 . The CEA doubling time was less than or equal to 6 months in 6 patients, ranged from 7 to 24 months in 18 , and was longer than 24 months in 27, among whom it was longer than 60 months in 13.

The calcitonin and CEA doubling times could both be determined in 50 patients, and were strongly correlated (Kendall correlation coefficient $=0.53, P<0.0001$ ). In the 24 patients with the calcitonin doubling time longer than 24 months, the CEA doubling time ranged from 3 to 146 months and was shorter or equal to 24 months in four patients. On the other hand, in the 26 patients with the calcitonin doubling time shorter or equal to 24 months, the CEA doubling time ranged from 2 to 61 months and was longer than 24 months in seven patients.

\section{MI and Ki-67 immunostaining}

MI and Ki-67 immunostaining were assessed for 33 patients on 8 primary tumors and 25 lymph node metastases. No mitosis was observed in 23 tumor samples, only one mitosis in 5 samples and two to fifteen mitoses $(2,3,6,7,15)$ in 5 samples. This low MI precluded the search for any relationship with the progression status.

The percentage of cells labeled with Ki-67 was equal to or less than 1 in 13 tumor samples, ranged from 2 to 5 in 7 , from 10 to 15 in 6 , and was equal to or above 20 (median 30, range 20-90) in 7.

\section{Relationships between studied parameters and progression status}

These parameters were then studied in the 45 patients with imaged disease, who could be classified as with 
either stable or progressive disease. Among these 45 patients, the calcitonin and CEA doubling times could both be determined in 40 patients, Ki-67 labeling index in 24 patients and SUV $_{\text {max }}$ in the 45 patients.

The calcitonin and CEA levels were not different between patients with either progressive or stable disease. The median calcitonin level was $1510 \mathrm{pg} / \mathrm{ml}$ (range $21-168000 \mathrm{pg} / \mathrm{ml}$ ) in patients with stable disease and $1564 \mathrm{pg} / \mathrm{ml}$ (range 191-247000 pg/ml) in patients with progressive disease $(P=0.44)$. For CEA, the median levels were $37 \mathrm{ng} / \mathrm{ml}$ (range 2-5845 ng/ml) and $109 \mathrm{ng} / \mathrm{ml}$ (range $1-3763 \mathrm{ng} / \mathrm{ml} ; \quad P=0.25$ ) respectively.

In patients with progressive disease, median calcitonin and CEA doubling times were 12 months (range 3-64 months) and 12 months (range 2-110 months) respectively; in those with stable disease, they were 48 months (range 13-107 months) and 58 months (range 13-100 months) respectively. The differences were highly significant $(P<0.0001)$, with very few overlaps between the two groups of patients (Fig. 3). Patients with concordant calcitonin and CEA doubling times could be reliably classified: among the 17 patients with both the calcitonin and CEA doubling times shorter than 25 months, 16 (94\%) had progressive disease, and among the 14 patients with both the calcitonin and CEA doubling times longer than 24 months, 12 (86\%) had stable disease. However, $9(20 \%)$ patients had discordant calcitonin and CEA doubling times, 4 with calcitonin doubling times longer than 24 months and CEA doubling times shorter than 25 months, and 3 (75\%) of them had progressive disease; 5 patients had a calcitonin doubling time shorter than 25 months but a CEA doubling time longer than 24 months, and 2 (40\%) had progressive disease (Table 1).

The MI and Ki-67 labeling indexes were not significantly associated with the progression of the disease (KruskalWallis test, $P=0.19$ for MI and $P=0.11$ for Ki-67). The median Ki-67 labeling index was 10 (range 0.5-60) in patients with progressive disease and 3 (range 0.5-10) in those with stable disease. Out of the 13 patients with Ki-67 labeling index $\leq 5$ and the 11 with Ki-67 labeling index $>5,8(62 \%)$ and $10(91 \%)$ patients respectively had progressive disease. Ki-67 labeling was associated with the calcitonin (Kendall correlation coefficient $=-0.36$, $P=0.03$ ) and CEA doubling times (Kendall correlation coefficient $=-0.51, P=0.002)$. The calcitonin doubling time was shorter than 25 months in 10 (91\%) patients with Ki-67 labeling higher or equal to 6 and in $6(55 \%)$ patients with Ki-67 labeling lower than 6. The CEA doubling time was shorter than 25 months in $9(90 \%)$ patients with Ki-67 labeling higher or equal to 6 and in 3 (27\%) patients with Ki-67 labeling lower than 6 (Table 2).

The median SUV was 4 (range 0-6) in patients with progressive disease, and 0 (range 0-3) in those with stable disease. The difference was highly significant $(P=0.0009)$, but there was a large overlap between the two groups of patients. The disease was progressive in 8
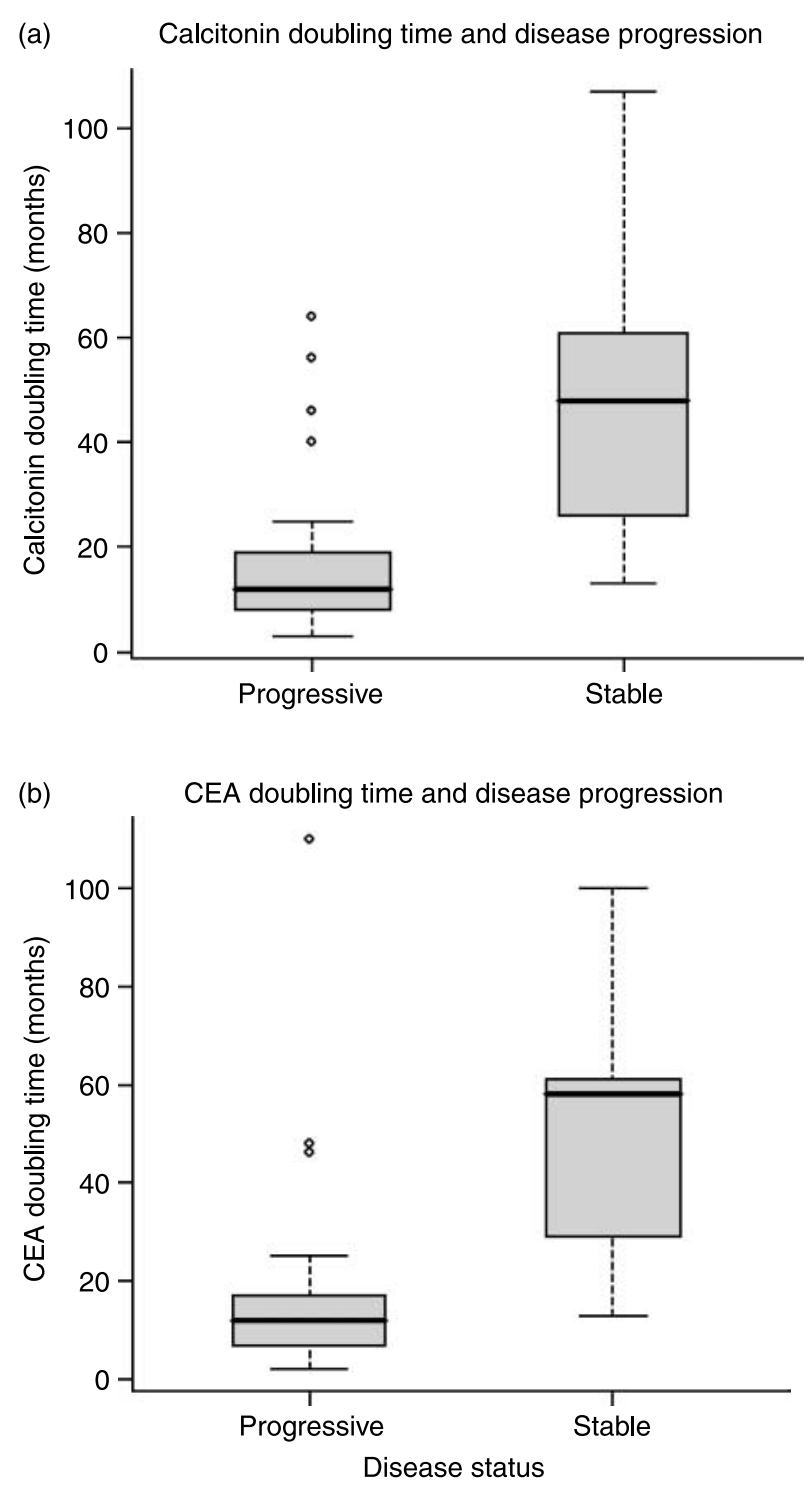

Figure 3 (a) Plasma calcitonin doubling time according to disease progression determined according to RECIST. The edges of the box are the 25th and 75th percentiles and the line in the box is the median. The ends of the vertical lines or 'whiskers' indicate the minimum and maximum data values, unless outliers (noted with circle) are present, in which case the whiskers extended to a maximum of 1.5 times the inter-quartile range. Calcitonin doubling time was available in 25 patients with progressive disease and in 17 patients with stable disease. It was significantly lower in patients with disease progression than in patients with stable disease $(P<0.0001)$. (b) Serum CEA doubling time according to disease progression, determined according to RECIST. The edges of the box are the 25th and 75th percentiles and the line in the box is the median. The ends of the vertical lines or 'whiskers' indicate the minimum and maximum data values; unless outliers (noted with circle) are present in which case the whiskers extended to a maximum of 1.5 times the inter-quartile range. CEA doubling time was available in 25 patients with progressive disease and in 17 patients with stable disease. It was significantly lower in patients with disease progression than in patients with stable disease $(P<0.0001)$. 
Table 1 Relationships between calcitonin (Ct) and carcinoembryonic antigen (CEA) doubling times (DT), standardized uptake value (SUV) determined on PET scan, mitotic index (MI), and Ki-67 labeling determined on tumor samples, and stable or progressive disease according to response evaluation criteria in solid tumors (RECIST).

\begin{tabular}{|c|c|c|c|}
\hline & Stable disease & Progressive disease & $P$ value \\
\hline Ct and CEA DT $\leq 24$ months & $1(6 \%)$ & $16(94 \%)$ & \\
\hline Ct DT $\geq 25$ months and CEA DT $\leq 24$ months & $1(25 \%)$ & $3(75 \%)$ & \\
\hline Ct DT $\leq 24$ months and CEA DT $\geq 25$ months & $3(60 \%)$ & $2(40 \%)$ & \\
\hline Ct and CEA DT $\geq 25$ months & $12(86 \%)$ & $2(14 \%)$ & $<0.0001^{a}$ \\
\hline $\mathrm{SUV}_{\max } \leq 2$ & $13(62 \%)$ & $8(38 \%)$ & \\
\hline SUV $_{\max }>2$ & $6(25 \%)$ & $18(75 \%)$ & $0.01^{\mathrm{b}}$ \\
\hline $\mathrm{MI}=0$ & $5(33 \%)$ & $10(67 \%)$ & \\
\hline $\mathrm{MI} \geq 1$ & $1(11 \%)$ & $8(89 \%)$ & $0.35^{\mathrm{a}}$ \\
\hline $\mathrm{Ki}-67 \leq 5$ & $5(38 \%)$ & $8(62 \%)$ & \\
\hline $\mathrm{Ki}-67 \geq 6$ & $1(9 \%)$ & $10(91 \%)$ & $0.17^{\mathrm{a}}$ \\
\hline
\end{tabular}

${ }^{a}$ Fisher exact test.

${ }^{\mathrm{b}} \chi^{2}$ test.

(38\%) out of the 21 patients with an SUV equal to or lower than 2 and in 18 (75\%) out of the 24 patients with an SUV higher than 2. SUV $\max$ was correlated with both the calcitonin (Kendall correlation coefficient $=-0.31$, $P=0.007)$ and CEA doubling times (Kendall correlation coefficient $=-0.32, P=0.007)$. The calcitonin doubling time was shorter than 25 months in 8 (42\%) out of the 19 patients with an SUV equal to or lower than 2 and in 16 $(70 \%)$ of the 23 patients with an SUV higher than 2 (Table 2). Finally, among the 9 patients with discordant calcitonin and CEA doubling times, progressive disease was observed in $2(40 \%)$ of the 5 patients with an SUV $\leq 2$ and in $3(75 \%)$ of the 4 patients with an SUV $>2$. The knowledge of SUV $\max$ did not improve the assessment of the progressive/stable disease status brought by the calcitonin and CEA doubling times.

\section{Discussion}

We studied tumor burden and progression status in a series of 55 consecutive MTC patients treated at a single institution. These patients may not reflect the general behavior of the MTC patients since in a tertiary referral center, patients with aggressive disease may be overrepresented. Indeed, among the 45 patients with demonstrated lesions, 26 had progressive disease.

A complete imaging study visualized neoplastic foci in all the patients with plasma calcitonin levels above $816 \mathrm{pg} / \mathrm{ml}$, in accordance with the previous studies (25), and higher levels were associated with larger numbers of tumor foci. However, there was a large overlap between the groups of patients, indicating that the production of calcitonin and CEA differs between tumors. Therefore, in an individual patient, higher calcitonin and CEA should increase the suspicion of distant metastases, but calcitonin and CEA determinations cannot replace imaging modalities.

Serum calcitonin and CEA levels were not related to progressive or stable status of the disease. In the previous studies on the MTC patients, the trends in the calcitonin and CEA levels have been related to clinical kinetic parameters (7-14), but no direct relationship with the progression rate assessed on repeated imaging modalities was reported. In the present study, the progressive or stable nature of the disease at an interval of 1 year was assessed using RECIST and this will permit decision on an objective basis of any therapeutic intervention, and to assess the effects of any therapy, in particular in the frame of early controlled trials; this will also permit less aggressive monitoring protocol in patients with long doubling times. The doubling times could be accurately determined by taking into account the measurements performed during long periods of time in the absence of any therapeutic interventions; this allows avoidance of biases due to spontaneous variations in the circulating calcitonin levels at short-term intervals, but implies a sufficiently long-term follow-up of patients in routine practice for months or even years. The progression status

Table 2 Relationships between calcitonin (Ct) and carcinoembryonic antigen (CEA) doubling times (DT), standardized uptake value (SUV) determined on PET scan, mitotic index (MI), and Ki-67 labeling determined on tumor samples.

\begin{tabular}{lcc}
\hline & $\begin{array}{c}\text { Ct DT } \leq \mathbf{2 4} \\
\text { months }\end{array}$ & Ct DT $\geq \mathbf{2 5}$ months \\
\hline CEA DT $\leq 24$ months & $19(73 \%)$ & $4(27 \%)$ \\
CEA DT $\geq 25$ months & $7(26 \%)$ & $20(74 \%)$ \\
SUV $_{\max } \leq 2$ & $8(42 \%)$ & $11(58 \%)$ \\
SUV $\max >2$ & $16(70 \%)$ & $7(30 \%)$ \\
$\mathrm{MI}=0$ & $10(67 \%)$ & $5(33 \%)$ \\
$\mathrm{MI} \geq 1$ & $6(86 \%)$ & $1(14 \%)$ \\
$\mathrm{Ki}-67 \leq 5$ & $6(55 \%)$ & $5(45 \%)$ \\
$\mathrm{Ki}-67 \geq 6$ & $10(91 \%)$ & $1(9 \%)$ \\
\hline & CEA DT $\leq \mathbf{2 4}$ & CEA DT $\geq 25$ \\
& months & months \\
\hline $\mathrm{SUV} \mathrm{m}_{\max } \leq 2$ & $8(44 \%)$ & $10(56 \%)$ \\
$\mathrm{SUV} \mathrm{max}_{\max }>2$ & $14(61 \%)$ & $9(39 \%)$ \\
$\mathrm{MI}=0$ & $6(43 \%)$ & $8(57 \%)$ \\
$\mathrm{MI} \geq 1$ & $6(86 \%)$ & $1(14 \%)$ \\
$\mathrm{Ki}-67 \leq 5$ & $3(27 \%)$ & $8(73 \%)$ \\
$\mathrm{Ki}-67 \geq 6$ & $9(90 \%)$ & $1(10 \%)$ \\
\hline
\end{tabular}


was found to be strongly related to the calcitonin and CEA doubling times, with very few overlaps between the two groups of patients. In the absence of therapeutic intervention, we did not observe patients in whom calcitonin or CEA doubling times changed during the study period.

The calcitonin and CEA doubling times were strongly correlated in $80 \%$ of patients, and in these patients they represent an excellent indicator of progressive or stable disease: $94 \%$ of patients with the doubling times shorter than 25 months had progressive disease and $86 \%$ of patients with the doubling times longer than 24 months had stable disease. It is interesting to note that the same cutoff of 24 months was reported in a previous study (11). The calcitonin and CEA doubling times were discordant in $20 \%$ of patients, resulting in non-parallel trends, and progressive disease was observed in five out of the nine patients with either calcitonin doubling times longer than 24 months and shorter CEA doubling times, or with calcitonin doubling times shorter than 25 months and with CEA doubling times longer than 24 months. Therefore, as described previously $(11,12)$, both the calcitonin and CEA doubling times should be determined and taken into account to predict the progression rate, as rapidly progressing tumors usually produce rapidly increasing amounts of both calcitonin and CEA, but also in a few cases relatively stable amounts of either calcitonin or CEA but increasing amounts of the other marker. It must be emphasized that even in patients with progressive disease, the doubling times of calcitonin and CEA were much longer than the doubling times of other markers in patients with cancers from other origins, but were similar to the PSA doubling time observed in patients with prostate carcinoma (28). Indeed, this is in accordance with the prolonged survival observed in the majority of the MTC patients with metastases, despite the absence of effective systemic treatment.

The other studied markers of progression cannot be used to assess progression status in individual patients. MI and Ki-67 labeling were low in the majority of tumors, in accordance with the long calcitonin and CEA doubling times. For this reason, MI or Ki-67 cannot be used as an indicator of tumor growth rate in contrast to what is routinely performed for other tumors $(29,30)$. Finally, SUV $_{\text {max }}$ was lower in patients with stable disease and long calcitonin and CEA doubling times than in those with progressive disease, but there was a large overlap between these two groups of patients.

In conclusion, the calcitonin and CEA levels reflect the tumor burden, and in the majority of patients their doubling times are closely related to tumor progression. Determination of MI or Ki-67 labeling index or SUV on FDG PET cannot substitute the direct determination of progression according to the RECIST or both the calcitonin and CEA doubling times.

\section{Acknowledgements}

The authors indicate no potential conflict of interest. This work was supported by Programme Hospitalier de Recherche Clinique AOM 02-118.

\section{References}

1 Pellegriti G, Leboulleux S, Baudin E, Bellon N, Scollo C, Travagli I \& Schlumberger M. Long-term outcome of medullary thyroid carcinoma in patients with normal postoperative medical imaging. British Journal of Cancer $2003 \mathbf{8 8}$ 1537-1542.

2 Leboulleux S, Baudin E, Travagli J \& Schlumberger M. Medullary thyroid carcinoma. Clinical Endocrinology $200461299-310$.

3 Collins V, Loeffler R \& Tivey H. Observations on growth rates of human tumors. American Journal of Roentgenology, Radium Therapy, and Nuclear Medicine $1956 \mathbf{7 6} 988-1000$.

4 Santoro M \& Carlomagno F. Drug insight: small-molecule inhibitors of protein kinases in the treatment of thyroid cancer. Nature Clinical Practice, Endocrinology and Metabolism 20062 $42-52$.

5 Giraudet AL, Vanel D, Leboulleux S, Aupérin A, Dromain C, Chami L, Ny Tovo N, Lumbroso J, Lassau N, Hartl D, Travagli JP, Baudin E, Bonniaud G \& Schlumberger M. Imaging medullary thyroid carcinoma with persistent elevated calcitonin levels. Journal of Clinical Endocrinology and Metabolism 200792 4185-4190.

6 Therasse P, Arbuck S, Eisenhauer E, Wanders J, Kaplan R, Rubinstein L, Verweij J, Van Glabbeke M, van Oosterom A, Christian M \& Gwyther S. New guidelines to evaluate the response to treatment in solid tumors. European Organization for Research and Treatment of Cancer, National Cancer Institute of the United States, National Cancer Institute of Canada. Journal of National Cancer Institute 200092 205-216.

7 Stepanas A, Samaan N, Hill CJ \& Hickey R. Medullary thyroid carcinoma: importance of serial serum calcitonin measurement. Cancer 197943 825-837.

8 Mendelsohn G, Wells SJ \& Baylin S. Relationship of tissue carcinoembryonic antigen and calcitonin to tumor virulence in medullary thyroid carcinoma. An immunohistochemical study in early, localized, and virulent disseminated stages of disease. Cancer 198454 657-662.

9 Cohen R, Campos JM, Salaun C, Heshmati HM, Kraimps JL, Proye C, Sarfati E, Henry JF, Niccoli-Sire P \& Modigliani E. Preoperative calcitonin levels are predictive of tumor size and postoperative normalization in medullary thyroid carcinoma. Journal of Clinical Endocrinology and Metabolism 200085 919-922.

10 Pyke C, Hay I, Goellner J, Bergstralh E, van Heerden J \& Grant C. Prognostic significance of calcitonin immunoreactivity, amyloid staining, and flow cytometric DNA measurements in medullary thyroid carcinoma. Surgery $1991 \mathbf{1 1 0} 964-970$ (discussion 970-961).

11 Barbet J, Campion L, Kraeber-Bodéré F \& Chatal J. Prognostic impact of serum calcitonin and carcinoembryonic antigen doubling-times in patients with medullary thyroid carcinoma. Journal of Clinical Endocrinology and Metabolism $2005906077-$ 6084.

12 Busnardo B, Girelli M, Simioni N, Nacamulli D \& Busetto E. Nonparallel patterns of calcitonin and carcinoembryonic antigen levels in the follow-up of medullary thyroid carcinoma. Cancer $198453278-285$.

13 Miyauchi A, Onishi T, Morimoto S, Takai S, Matsuzuka F, Kuma K, Maeda M \& Kumahara Y. Relation of doubling time of plasma calcitonin levels to prognosis and recurrence of medullary thyroid carcinoma. Annals of Surgery 1984199 461-466.

14 Rougier P, Calmettes C, Laplanche A, Travagli J, Lefevre M, Parmentier C, Milhaud G \& Tubiana M. The values of calcitonin 
and carcinoembryonic antigen in the treatment and management of nonfamilial medullary thyroid carcinoma. Cancer $1983 \mathbf{5 1}$ 855-862.

15 Katoh R, Bray C, Suzuki K, Komiyama A, Hemmi A, Kawaoi A, Oyama T, Sugai T \& Sasou S. Growth activity in hyperplastic and neoplastic human thyroid determined by an immunohistochemical staining procedure using monoclonal antibody MIB-1. Human Pathology 199526 139-146.

16 Saltman B, Singh B, Hedvat C, Wreesmann V \& Ghossein R. Patterns of expression of cell cycle/apoptosis genes along the spectrum of thyroid carcinoma progression. Surgery $2006 \mathbf{1 4 0}$ 899-905 (discussion 905-896).

17 Tisell L, Oden A, Muth A, Altiparmak G, Mõlne J, Ahlman H \& Nilsson O. The Ki67 index a prognostic marker in medullary thyroid carcinoma. British Journal of Cancer 200389 2093-2097.

18 Robbins R, Wan O, Grewal R, Reibke R, Gonen M, Strauss H, Tuttle R, Drucker W \& Larson S. Real-time prognosis for metastatic thyroid carcinoma based on 2-[18F]fluoro-2-deoxy-D-glucosepositron emission tomography scanning. Journal of Clinical Endocrinology and Metabolism 2006 91 498-505.

19 Downey R, Akhurst T, Gonen M, Vincent A, Bains M, Larson S \& Rusch V. Preoperative F-18 fluorodeoxyglucose-positron emission tomography maximal standardized uptake value predicts survival after lung cancer resection. Journal of Clinical Oncology 200422 3255-3260.

20 Leboulleux S, Schroeder P, Schlumberger M \& Ladenson P. The role of PET in follow-up of patients treated for differentiated epithelial thyroid cancers. Nature Clinical Practice, Endocrinology and Metabolism 20073 112-121.

21 Schwartz D, Rajendran J, Yueh B, Coltrera M, Leblanc M, Eary J \& Krohn K. FDG-PET prediction of head and neck squamous cell cancer outcomes. Archives of Otolaryngology - Head and Neck Surgery 2004130 1361-1367.

22 Vansteenkiste J, Stroobants S, Dupont P, De Leyn P, Verbeken E, Deneffe G, Mortelmans L \& Demedts M. Prognostic importance of the standardized uptake value on (18)F-fluoro-2-deoxy-glucosepositron emission tomography scan in non-small-cell lung cancer: an analysis of 125 cases. Leuven Lung Cancer Group. Journal of Clinical Oncology 199917 3201-3206.

23 Sperti C, Pasquali C, Chierichetti F, Ferronato A, Decet G \& Pedrazzoli S. 18-Fluorodeoxyglucose positron emission tomography in predicting survival of patients with pancreatic carcinoma. Journal of Gastrointestinal Surgery 20037 953-959 (discussion 959-960).

24 Adams S, Baum R, Hertel A, Schumm-Dräger P, Usadel K \& Hör G. Metabolic (PET) and receptor (SPET) imaging of well- and less welldifferentiated tumours: comparison with the expression of the Ki67 antigen. Nuclear Medicine Communications 199819 641-647.

25 Ong S, Schöder H, Patel S, Tabangay-Lim I, Doddamane I, Gönen M, Shaha A, Tuttle R, Shah J \& Larson S. Diagnostic accuracy of 18F-FDG PET in restaging patients with medullary thyroid carcinoma and elevated calcitonin levels. Journal of Nuclear Medicine $2007 \mathbf{4 8} 501-507$.

26 Motté P, Vauzelle P, Gardet P, Ghillani P, Caillou B, Parmentier C. Bohuon C \& Bellet D. Construction and clinical validation of a sensitive and specific assay for serum mature calcitonin using monoclonal anti-peptide antibodies. Clinica Chimica Acta 1988 174 35-54.

27 AJCC. Thyroid. In American Joint Committee on Cancer: AJCC Cancer Staging Manual, edn 6, pp 77-87 New York: Springer, 2002.

28 Tollefson M, Slezak J, Leibovich B, Zincke H \& Blute M. Stratification of patient risk based on prostate-specific antigen doubling time after radical retropubic prostatectomy. Mayo Clinic Proceedings 200782 422-427.

29 Bertolini F, Bengala C, Losi L, Pagano M, Iachetta F, Dealis C, Jovic G, Depenni R, Zironi S, Falchi AM, Luppi G \& Conte PF. Prognostic and predictive value of baseline and posttreatment molecular marker expression in locally advanced rectal cancer treated with neoadjuvant chemoradiotherapy. International Journal of Radiation Oncology, Biology, Physics 200768 1455-1461.

30 de Azambuja E, Cardoso F, de Castro GJ, Colozza M, Mano M, Durbecq V, Sotiriou C, Larsimont D, Piccart-Gebhart M \& Paesmans M. Ki-67 as prognostic marker in early breast cancer: a meta-analysis of published studies involving 12155 patients. British Journal of Cancer 200796 1504-1513.

Received 11 November 2007

Accepted 13 November 2007 